\title{
Nahrungsmittel- Unverträglichkeit?
}

Ein einfacher Test kann Aufschluss bringen. Gespräch mit Dr. Johanna Helm, Ärztin für Allgemeinmedizin in Wien

Nahrungsmittel-Unverträglichkeiten sind in aller Munde. Kann man diese auch diagnostisch erfassen? - Wir sprachen mit Dr. Johanna Helm, die sich seit einiger Zeit mit betroffenen Patienten und dem Testverfahren ImuPro 300 befasst.

Promed komplementär: Wie oft wenden sich Patienten mit einem Verdacht auf eine Nahrungsmittelunverträglichkeit an Sie?

HELM: Es wenden sich häufig Patienten mit chronischen Erkrankungen oder Beschwerden an mich, die bereits viele Untersuchungen und Therapien hinter sich haben, aber keine zufriedenstellende Besserung erfahren haben. Im Laufe meines ärztliches Gespräches und der Befunddurchsicht ergibt sich dann immer wieder der Verdacht auf eine Nahrungsmittelunverträglichkeit. Manche Patienten kommen schon gezielt mit diesem Verdacht, da sie selbst beobachtet haben, dass sich ihre Beschwerden nach dem Genuss bestimmter Lebensmittel einstellen.

Welche Beschwerdebilder geben die Patienten anamnestisch an?

HELM: Nahrungsmittelunverträglichkeiten rufen die verschiedensten Beschwerdebilder hervor. Von den Symptomen kann der erfahrene Arz bereits auf die Art der Unverträglich-

keit schließen. Wir unterscheiden hier Unverträglichkeiten auf Milchoder Fruchtzucker, andererseit kann eine Histaminintoleranz vorliegen, eine Typ I (Sofort-)Allergie oder eben eine IgG vermittelt Typ 3-Allergie, der ein immunologi scher Mechanismus zugrunde lieg und die mit dem ImuPro 300 Test diagnostiziert werden kann. Speziel bei der letztgenannten Form geben die Patienten oft Verdauungsbeschwerden, rezidivierende Diarrhoen und Blähungen an, sowie auch Migräne, Gelenksbeschwerden, Hauterkrankungen, Übergewich und chronische Müdigkeit, um nu einige zu nennen.

Finden Sie bei diesen Patienten klinisch allein funktionelle Störungen oder gibt es organische Erkrankunoder, welche im Zus gen, welche im Zusammenhang $\mathrm{m}$ Nahrungsmittelunverträglichkeiten auftreten können?

HELM: Eine fehlerhafte Immunantwort gegenüber Lebensmittelantigenen kann die Ursache für Immunpathologien, wie entzündliche Erkrankungen des Dünndarmes ode Autoimmunerkrankungen sein. Di erhöhte Permeabilität der Ileum schleimhaut und die chronisch entzündliche Aktivität sind Wegbereiter für organische Erkrankungen: rheumatische Arthritis, chronisch Rhinitis und Sinusitis, Ekzeme, atopische Dermatitis, Morbus Crohn

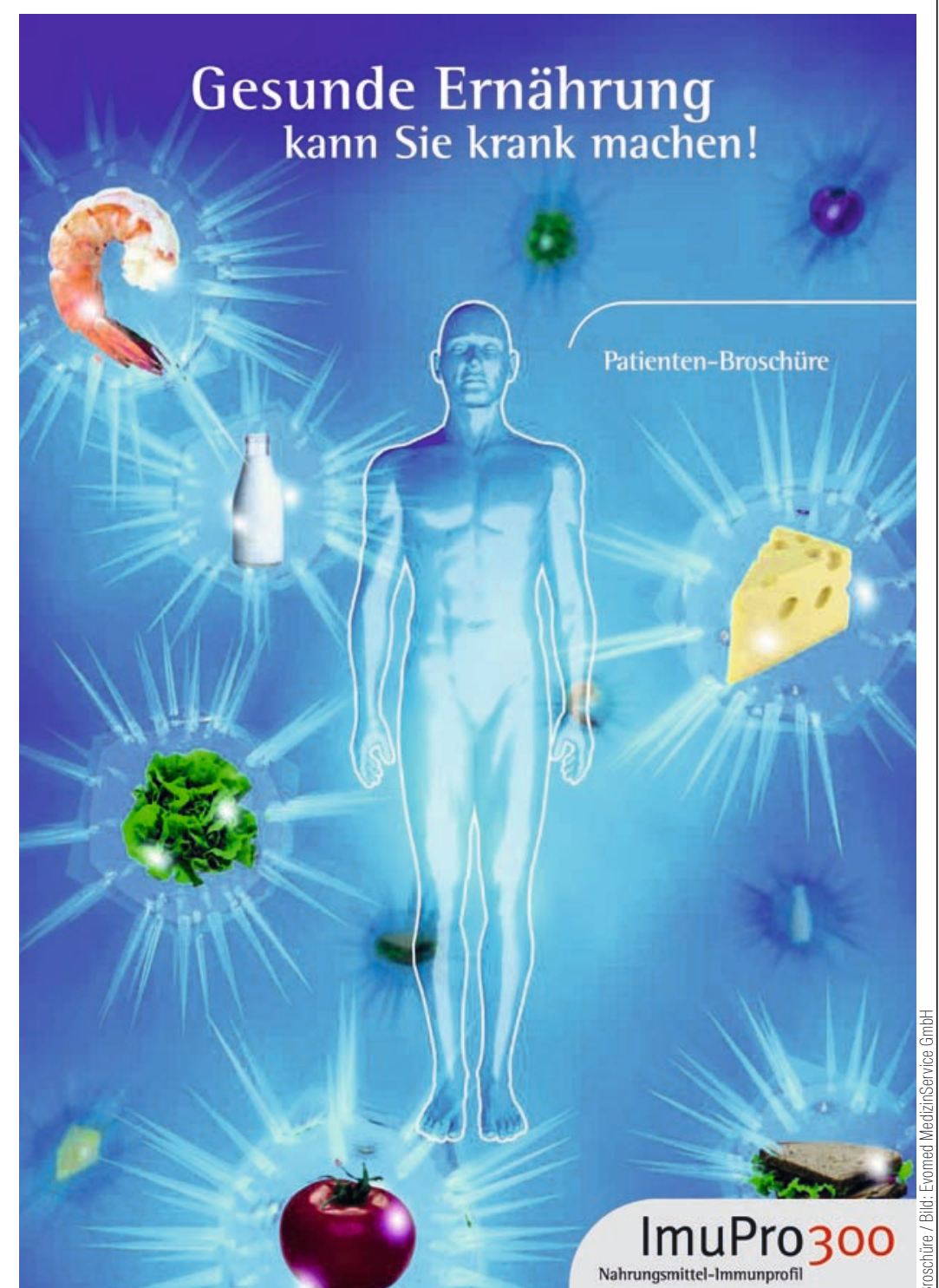

Die Patienten-Broschüre zum Testverfahren ImuPro 300
Stoffwechselstörungen wie Diabetes mellitus II, Hypertonie, um nur einige zu nennen.

Welche Rolle kommt dem Hausarzt m Rahmen der Abklärung eines Verdachts auf Nahrungsmittelallergie zu?

HELM: Dem Hausarzt/der Hausärztin kommt eine sehr wichtige Rolle zu, da er/sie zumeist der erste Ansprechpartner und mit der Krankengeschichte des Patienten vertraut ist. Da gemäß seriöser Studien mit eine Prävalenz der Nahrungsmittelunverträglichkeiten von mindestens 20 Prozent ausgegangen werden kann, is die Wahrscheinlichkeit, dass solche Intoleranzen bei den verschiedensten Beschwerdebildern eine Rolle spielen, sehr hoch. Es lohnt sich für Allgemeinmediziner meiner Meinung nach sehr, über dieses Thema gut informiert zu sein, da man zwischen den unterschiedlichen Unverträglichkeiten, wie vorhin erwähnt unterscheiden muss. Da anzuneh men ist, dass die Nahrungsmittelunverträglichkeiten in der Bevölkerung zunehmen werden, sind Hausärzte in Zukunft sicher noch mehr gefordert, sich mit dieser Diagnostik und de entsprechenden Ernährungsberatung auseinander zu setzen.

Wie sind Sie auf das Testverfahren ImuPro 300 gestoßen?

HELM: Ich habe vor beinahe zehn ahren erstmals von diesem Testverfahren ImuPro 300 gehört, damal durch einen bekannten Biochemike der den Test nach Österreich gebracht hat. Mein Interesse daran war von Anfang an groß, da ich schon damals be manchen Patienten den dringenden Verdacht auf eine Nahrungsmittelintoleranz hatte, mit den damals zur Verfügung stehenden Untersuchungsmöglichkeiten aber nicht im mer zur richtigen Diagnose kam. Die Fructose- und Lactoseintoleranz konnte man mit dem $\mathrm{H} 2$ Atemtest untersuchen, und die Histaminintoleranz konnte in einem Bluttest festgestellt werden, aber damit konnte man eben noch nicht alle Unverträglichkeiten aufdecken.

Bitte erklären Sie das Testverfahren in kurzen Worten.

HELM: Der Test ist ein ELISA Verfah ren, bei dem spezifische IgG Antikörper aus dem Blut quantitativ bestimmt werden. Dazu ist nur eine kleine Menge Venenblut erforderlich. Wir können damit bis zu $273 \mathrm{Le}$ bensmittel und Zusatzstoffe untersuchen. Mit einem Vor-Screening test, bei dem einige häufig unverträgliche Nahrungsmittel getestet werden, kann die Notwendigkeit, einen erweiterten Test zu empfehlen, aufzeigen. Nach der photometrischen Auswertung wird die nachgewiesene IgG-Aktivität entsprechend dem Grad der Unverträglichkeit zwischen den Stufen 1 und 4 ausgewertet. Der Labortest wird in Österreich

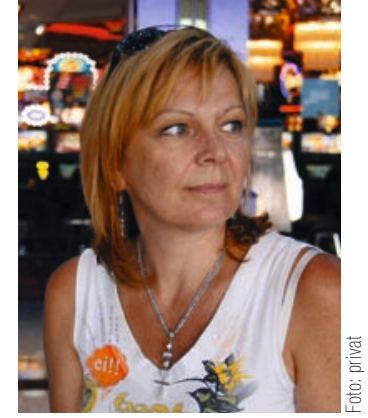

Dr. Johanna Helm Ärztin für Allgemeinmedizin und Facharztin für Arbeitsmedizin, Wien Ordination Pumperlgsu

„Es wenden sich häufig Patienten mit chronischen Erkran-

kungen oder Beschwerden an mich, die bereits viele Untersuchungen und erfolglose 'Therapien hinter sich haben."

nur im Labor Dr. Dostal, 1190 Wien, durchgeführt. Da die Antikörper stabil und unempfindlich sind, kan die Blutproben per Post aus ganz Österreich an das Labor gesendet werden. Der Befund wird dann in einem ausführlichen ärztlichen Gespräch, das ich für sehr wichtig halte, dem Patienten vermittelt und ein individueller Ernährungsplan zusammengestellt. Je nach dem Stärkegrad de Unverträglichkeit sollen dann die betroffenen Nahrungsmittel für eine bestimmte Zeit aus dem Speiseplan gestrichen werden.

Gibt es wissenschaftliche Daten, die das Verfahren stützen?

HELM: Mir sind wissenschaftliche Studien bekannt, die die Bedeutung der Analyse von Nahrungsmittel-Allergien Typ III bei Morbus Crohn, sowie bei Migräne eindeutig belegen Besonders interessant für mich als praktische Ärztin sind auch die Ergebnisse der in Deutschland bei ca. 1000 Patienten durchgeführten Anwendungsbeobachtung, die deutliche Verbesserungen bei Verdauungsproblemen, Hautkrankheiten un Migräne zeigen.

Wie ist die weitere Betreuung de getesteten Patienten organisiert? HELM: Wie erwähnt, ist das ärztlich Gespräch mit dem Patienten seh wichtig, um die Bedeutung des Befundes und die weitere Ernährungsweise zu erklären. Es sollen ja durch die Ernährungsumstellung keinesfalls Nährstoffdefizite entstehen. Die Testpersonen erhalten außerdem seh ausführliche Unterlagen mit den wichtigen Informationen auch zum Nachlesen und ein persönliches Rezept- buchmit vielen Anregungen, um weiter genussvoll und ausgewogen speisen zu können. Ich empfehle nach etwa drei Monaten der Ernährungsumstellung einen weiteren Gesprächstermin, bei dem der Gesundheitszustand evaluiert und die weitere Vorgangsweise besprochen wird.

Welche Erfahrungen haben Sie in Ihrer Ordination mit dem Testverfahren gemacht?

HELM: Ich habe mit dem Test bishe nur die besten Erfahrungen gemacht, und ich überblicke mittlerweile einige Hundert Testergebnisse. Die Langzeiterfolge sind verblüffend, obwohl dies eine Therapie ohne jede Nebenwirkung ist! Manchmal ist die Ernährungsumstellung anfangs gar nicht so leicht umsetzbar, es müssen ft Ersatzlebensmittel besorgt werden, manches schmeckt ungewohnt und man muss eventuell mehr selbst kochen und sich mit der Ernährung einfach mehr beschäftigen. Aber ich kann behaupten, dass nahezu alle meine Patienten diese Unannehmlichkeiten gerne in Kauf nehmen, da sie schon nach kurzer Zeit einen positiven Effekt bemerken. Verdauungsprobleme verschwinden zumeis schon nach einer Woche der Ernährungsumstellung, andere Beschwerden bestehen manchmal einige Wochen weiter, aber alle Patienten be richten zumindest über eine deutliche Besserung, und nach drei Monaten sind bei vielen die Symptome völlig verschwunden und es können Medikamente reduziert oder sogar ganz abgesetzt werden.

Sind Weiterentwicklungen des Testverfahrens zu erwarten?

HELM: Die Imupro-Tests werden laufend weiterentwickelt. Der ursprüngliche Imupro 300 Test wurde durch den Screening, sowie Imupro 100 und 200 ergänzt, so dass es möglich wurde, den für den jeweiligen Patienten geeigneten Test auszuwählen. Weiters wird die Auswahl der getesteten Lebensmittel laufend überarbeitet und ergänzt. Auch gibt sich das Labor viel Mühe, durch Teilnahme an Ringversuchen die Genauigkeit der Analysen zu optimieren.

Vielen Dank für das interessante Gespräch!

Informationen: www.imupro.de Kontakt zu Imupro-österreich: www.imupro.at E-Mail: imupro300@gmx.a

Korrespondenz:

Dr. Johanna He
Thimiggasse 17

1180 Wien

Tel.: +43/1/470 7799

Mit freundlicher Unterstützung der Helmconsulting KG.

SpringerMedizin.at

Weitere Informationen unter:

www.SpringerMedizin.at/

www.SpringerMedizin.at 